\title{
A rheological investigation of the association between a non- ionic microemulsion and hydrophobically modified PEG. Influence of polymer architecture
}

\author{
Filipe E. Antunes ${ }^{\mathrm{a}, \mathrm{b}}$, Krister Thuresson ${ }^{\mathrm{b}}$, Björn Lindman ${ }^{\mathrm{a}, \mathrm{b}}$, \\ Maria G. Miguel $^{\mathrm{a}, *}$ \\ a Department of Chemistry, Coimbra University, 3004-535 Coimbra, Portugal \\ ${ }^{\mathrm{b}}$ Physical Chemistry 1, Center for Chemistry and Chemical Engineering, Lund University, P.O. Box 124, SE-221 00 Lund, Sweden
}

Received 15 April 2002; accepted 10 September 2002

\begin{abstract}
Hydrophobically modified polymers (HM-P) typically behave as thickeners in a wide range of systems. The thickening effect in an aqueous solution of this kind of polymer depends on intermolecular hydrophobic associations and also on chain entanglements if the polymer concentration is significantly above the overlap concentration. In the present investigation a rather short end-capped polymer has been investigated at concentrations that are significantly below the overlap concentration. Despite the rather low polymer concentration, polymer chains were connected into a three-dimensional network by using microemulsion droplets as cross-linking points. The simple structure of the solution simplifies interpretations of results since chain entanglements can be expected to be of low importance and only intermolecular hydrophobic associations have to be considered. In particular the rheological response is in most cases well characterized by one single relaxation time and, then, the solution can be rationalized within the framework of the Maxwell model. We have found that the length of the polymer chain's hydrophobic end-groups, as well as the temperature, have a large influence on dynamics of the system, while the length of the hydrophilic mid-block has a relatively small significance. On the other hand, the connectivity in the system depends critically on the microemulsion concentration. Thus, a maximum was found in viscosity as a function of volume fraction, interpreted as being due to a decrease in crosslink lifetime.
\end{abstract}

(C) 2002 Elsevier Science B.V. All rights reserved.

Keywords: Hydrophobically modified polymers; Microemulsions; Rheology; Thickening; Viscosity

\section{Introduction}

Hydrophilic (homo)polymers have traditionally found use as rheology modifiers in water based systems. Since this type of polymers often is

* Corresponding author. Fax: +351-239-82-7703

E-mail address: mgmiguel@ci.uc.pt (M.G. Miguel). nonadsorbing to surfaces of colloids, a complica- 
tion frequently encountered is phase separation into one phase enriched in polymer and one phase concentrated in the colloids. This was one reason to why hydrophobically modified polymers (HMP), or water-soluble associative polymers (WSAP), appeared on the market some 15 years ago. The idea was that the amphiphilic properties of these polymers should increase compatibility with dispersions by adsorbing to the surfaces of colloidal particles, at the same time as they offer the possibility to form physically cross-linked networks via the hydrophobic moieties of the HM-P chains. The 'thickening effect' was expected to be high for this type of polymer, and it was anticipated that significantly smaller quantities were needed to obtain a desired shear profile as compared with traditional thickeners. However, it was soon realized that the physically crosslinked network was sensitive to other components in the solution and that the hydrophobic attractions appeared to be responsible also to other problems. For instance, instead of the desired increase in stability, phase separation may be induced due to bridging-related mechanisms.

Despite draw-backs that were encountered, HM-Ps are today found in many technical formulations [1]. Such formulations generally have in common a complex composition, and they may contain a range of liquids, particles and colloids, and surface-active compounds. Water based paints may serve as a representative example. To be able to understand underlying mechanisms that determine a sometimes unwanted behavior, investigated mixtures have to be simple and the number of components reduced. In the present investigation we have used a well characterized system that is based on a thoroughly investigated microemulsion [2], and rather mono disperse end-capped poly(ethylene glycol) polymers. The HM-P possibility to act as bridges between the microemulsions can strongly affect the system properties [3-5]. In the temperature and compositional range we have chosen to work (within $17{ }^{\circ} \mathrm{C}<T<27{ }^{\circ} \mathrm{C}$ and $5 \%<W(\mathrm{~S}+\mathrm{O})<20 \%)$, where $W(\mathrm{~S}+\mathrm{O})$ represents the weight percentage of (surfactant + oil), the microemulsion forms spherical droplets with a diameter of approximately $160 \AA$ [6]. The Fig. 1 is a schematic representation of the microemulsion

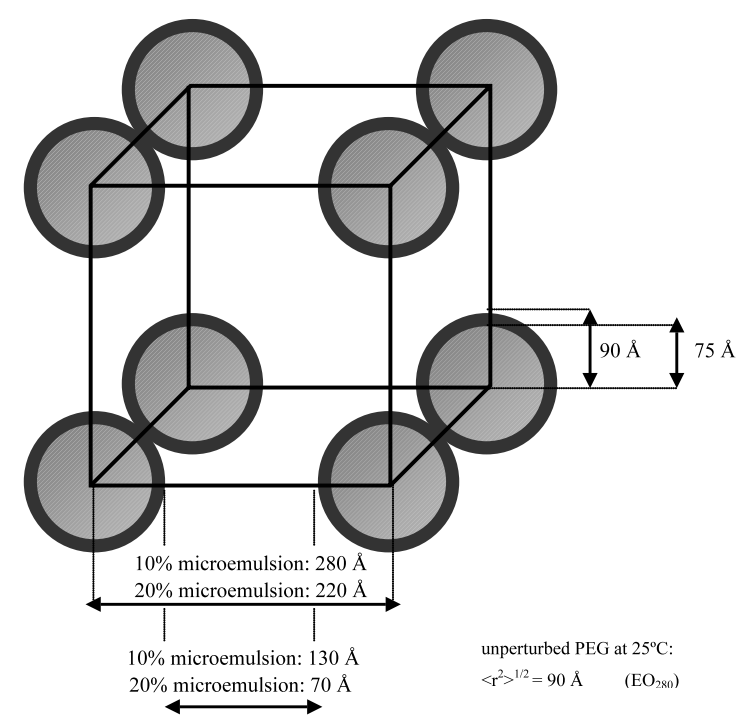

Fig. 1. Schematic representation of the $\mathrm{C}_{12} \mathrm{E}_{5}$-water-decane microemulsion system.

$\mathrm{C}_{12} \mathrm{E}_{5}$-decane-water system. To refer to the case of a water based paint formulation the microemulsion droplets may represent the binder particles (latex) and the end-capped polymer the thickener. In a simplified picture this structure can be viewed as a 'balls and stick' model because the polymerchains are bifunctional (or sometimes, as we will see below, only monofunctional). A reason to why interpretations become particularly simple in the present system is that the polymer concentration can be reduced well below the overlap concentration, and still retain a three-dimensional network that gives rise to a large rheological response. We have investigated effects of varying the hydrophobic end-group as well as of a variation of the length of the mid-block of the polymers, and of microemulsion concentration. Questions that we seek answers to are how microstructure and dynamics are influenced by these variations, and to obtain such information we have performed rheological investigations. In particular it was found that the rheological behavior is strongly dependent on the chemical composition of the polymer chains, and that a variation due to an incomplete synthesis may largely influence the results. Counterintuitively we found that above a certain microemulsion concentration the viscosity 
actually decreased with increasing concentration, keeping the number of polymer chains per microemulsion droplet fixed.

At higher microemulsion concentrations a very strong temperature dependency of the viscosity was found. These findings were attributed to that when microemulsion droplets approach, stress relaxation is facilitated since polymer-end groups can more easily move from one microemulsion droplet to another. This activation energy (from Arrhenius' plots) is strongly dependent on the concentration, a fact which is at first surprising, but we believe that this observation can be understood by recognizing that microemulsion droplets stabilized with EO-containing surfactants become less repulsive and may also attract each other at elevated temperatures.

Maxwell model was used to describe the dynamic moduli as a function of frequency. Within the framework of this model, that has one elastic component (spring) and one viscous component (dashpot) in series, the storage, $G^{\prime}$, and loss, $G^{\prime \prime}$, moduli become

$$
\begin{aligned}
& G^{\prime}(\omega)=G_{\infty} \frac{\tau^{2} \omega^{2}}{1+\tau^{2} \omega^{2}} \\
& G^{\prime \prime}(\omega)=G_{\infty} \frac{\tau \omega}{1+\tau^{2} \omega^{2}}
\end{aligned}
$$

$G_{\infty}$ is the plateau value of $G^{\prime}$ at high angular frequencies, $\omega=2 \pi f$, and $\tau$ is the characteristic time of the relaxation process. The fact that this simple model describes the data quite well suggests that the relaxation process, in the terminal zone, can be characterized by a single exponential relaxation time. Fitting Eqs. (1a) and (1b) to the experimental data gives values of $G_{\infty}$ and $\tau$ for each composition and temperature that was investigated. $G_{\infty}$ is expected to increase with concentration since it is proportional to the number density, $n$, of rheological active chains [7]

$G_{\infty} \cong n k_{\mathrm{B}} T$

where $k_{\mathrm{B}}$ is the Boltzmann factor and $T$ the absolute temperature. The characteristic time, $\tau$, of the relaxation process is related to the activation energy, $E$, for the relaxation process. $\tau \propto \exp \left(\frac{E}{k_{\mathrm{B}} T}\right)$

From Arrhenius' plots, which use the temperature dependency of $\tau, E$ can be obtained.

It can be mentioned that since $E$ values could be extracted, and therefore, the structure seems to be unaffected by temperature, it was possible to perform a superposition of mechanical spectra from different temperatures into one 'masterplot' by using the shift factors in Eqs. (4a) and (4b).

$$
\begin{aligned}
& a_{T}=\exp \left(-\frac{E}{k_{\mathrm{B}}}\left(\frac{1}{T_{0}}-\frac{1}{T}\right)\right) \\
& b_{T}=\frac{n\left(T_{0}\right) k_{\mathrm{B}} T_{0}}{n(T) k_{\mathrm{B}} T}=\frac{G_{\infty}\left(T_{0}\right)}{G_{\infty}(T)}
\end{aligned}
$$

\section{Experimental}

\subsection{Materials}

A range of hydrophobically end-modified polyethylene glycols (HM-PEG), that have been synthesized by a condensation between poly(ethylene glycol), PEG, and fatty alcohols, were received as kind gifts from Akzo Nobel Surface Chemistry Stenungsund, Sweden. The average molecular weights of the PEG polymers that were used was either 12000 or 20000 . This corresponds to 280 or 450 repeating oxyethylene units in the mid-block, respectively. Also two different alcohol samples were used in the preparation of the HM-PEG samples. Their hydrocarbon part was either a mixture of $\mathrm{C}_{20}-\mathrm{C}_{24}$ chains, or a mixture of $\mathrm{C}_{20}-\mathrm{C}_{28}$ chains, with average compositions of $\mathrm{C}_{21}$ and $\mathrm{C}_{24}$, respectively. Fig. 2 shows the molecular structure of the entities used.

Data for the four different HM-PEG polymers that were obtained by combining these substances are summarized in Table 1 . The condensation reaction will always result in a distribution between PEG polymers that have been di-substituted, mono-substituted, or polymers that have not been substituted at all. Such variations will of 


$$
\text { (2) }
$$

Fig. 2. Chemical structure of polymers and surfactant used.

course affect the properties of their solutions. Non-substituted PEG in these solutions is not expected to associate either to diblocks, to triblocks or to microemulsion droplets once the repulsion is expected and the PEG will reside in the aqueous phase in-between the droplets (mainly working as solvent).

Therefore, we have checked the polymer functionality by ${ }^{1} \mathrm{H}-\mathrm{NMR}$ (see below).

The microemulsion with which the different HM-PEG polymers were mixed is composed of nonionic surfactant pentaethylene oxide dodecyl ether $\left(\mathrm{C}_{12} \mathrm{EO}_{5}\right)$, water and decane at a constant surfactant to oil ratio of 52/48. $\mathrm{C}_{12} \mathrm{EO}_{5}$ was obtained from Nikko Chemicals Co. Ltd., Tokyo, Japan, and decane (>99\%) was obtained from Sigma.

Except for the composition analysis (with ${ }^{1} \mathrm{H}$ NMR), all chemicals were used without further purification and the water used had been passed through a Millipore ${ }^{\mathrm{TM}}$ purification unit.

\subsection{Methods}

To be able to prepare the mixtures containing HM-PEG and the microemulsion the following route had to be followed. First the HM-PEG polymer was dissolved in $\mathrm{C}_{12} \mathrm{EO}_{5}$, which is liquid at room temperature. To this solution an appropriate amount of water was added. This resulted in low-viscous aqueous solutions since surfactant micelles at this stage are numerous, and each polymer end-group can be decorated with a micelle. This is known to effectively reduce the viscosity in solutions of HM-P. To this lowviscous solution the appropriate amount of decane was added. The oil will enter the core of the micelles and increase their size. During this process the number of micelles will be significantly reduced. Actually, when oil is added the number of polymer molecules per microemulsion droplet or micelle $(\alpha)$ increases by roughly a factor of 25 (from about $\alpha=0.4-10$ ). This ratio has been kept constant throughout the investigation and in all samples that have been investigated $\alpha=10$. It should be noted that all samples have been prepared by weight and by assuming that all HM-PEG polymers were di-substituted. However, as we will see below, the effective $\alpha$ may be significantly smaller due to incomplete synthesis. To finalize the mixing of the samples a route was employed where the viscosity was temporarily reduced. When the temperature is increased to above $30{ }^{\circ} \mathrm{C}$ the phase behavior changes from spherical microemulsion droplets to a lamellar phase in equilibrium with a water-rich phase. This reduces the viscosity significantly and facilitates mixing of the samples (with a vortex). The microemulsion phase was again induced by lowering the temperature, and the mixing was com-

\begin{tabular}{|c|c|c|c|c|}
\hline Schematic structure and abbreviation & $\mathrm{C}_{21} \mathrm{EO}_{280} \mathrm{C}_{21}$ & $\mathrm{C}_{24} \mathrm{EO}_{280} \mathrm{C}_{24}$ & $\mathrm{C}_{21} \mathrm{EO}_{450} \mathrm{C}_{21}$ & $\mathrm{C}_{24} \mathrm{EO}_{450} \mathrm{C}_{24}$ \\
\hline Average composition of hydrophobes, by weight & $\mathrm{C}_{21}$ & $\mathrm{C}_{24}$ & $\mathrm{C}_{21}$ & $\mathrm{C}_{24}$ \\
\hline Approximate average molecular weight $(\mathrm{kDa})$ & 13 & 13 & 21 & 21 \\
\hline Approximate average end-end distance of unperturbed PEG $(\AA)$ (from [12]) & 90 & 90 & 115 & 115 \\
\hline
\end{tabular}
pleted by that the samples were centrifuged

Table 1

Shows data for the four different polymers that were used in the present investigation 
repeatedly in reversed directions and equilibrated in room temperature for at least $24 \mathrm{~h}$ before any measurements were performed.

The rheological measurements were performed by using a Physica UDS 200 rheometer or a Reologica StressTech rheometer. Both rheometers were equipped with automatic gap setting. A $5 \mathrm{~cm}$, $1^{\circ}$ cone and plate geometry with a solvent trap was used to all investigated samples, and the temperature in the measuring geometry was controlled to within $\pm 0.1{ }^{\circ} \mathrm{C}$ by a Peltier system. All measurements were performed with the instrument in the oscillatory shear mode, and to ensure that all determinations were performed in the linear viscoelastic regime, each rheological determination was preceded by stress-sweep measurements.

Before analyzing the composition of the HMPEG polymers by ${ }^{1} \mathrm{H}-\mathrm{NMR}$ they were purified according to the following procedure. The dry polymer powder was allowed to swell, or partly dissolve, in acetone for a few hours followed by addition of hexane. The less polar solvent precipitated the polymer as a white powder. This procedure was repeated five times, after which the polymer sample was dried thoroughly under vacuum, and later dissolved in $\mathrm{CDCl}_{3}$ to a concentration of $2 \mathrm{wt} . \%$. From the ratio of the peak area of the two different types of methylene groups, in the polymer hydrocarbon tails and in the oxyethylene part, it was found that the $\mathrm{C}_{y} \mathrm{EO}_{x} \mathrm{C}_{y}$ polymers all have an approximate substitution degree of about 1.2 (instead of the ideal 2.0). This may give an effective concentration of di-substituted HM-PEG polymers varying in between 20 and $60 \%$ of that expected if all HMPEG chains had been di-substituted. This will, as will be obvious below, have large implications to the behavior.

\section{Results and discussion}

The microemulsion that we have chosen to work with is well characterized, and spherical microemulsion droplets form in the concentration and temperature ranges that we have used $[2,6]$. The formed droplets can be viewed as oil-swollen micelles and they have a hydrocarbon core radius of about $75 \AA$. From an earlier investigation it is also known that the size and structure of the droplets are virtually insensitive to HM-PEG polymers [8]. Due to hydrophobic attraction a HM-PEG polymer is expected to adsorb to the microemulsion droplets via the end-groups, while the mid-block is expected to be in an aqueous environment. These requirements are fulfilled in a situation where polymer chains form loop conformations and both stickers from a HM-PEG polymer are attached to the same microemulsion droplet. This is likely to be the case when the average distance between microemulsion droplets is large compared with the length of the PEGblock of the polymers. When the average distance between micelles is in the same order, or small, as the length of the mid-block of the polymer chains the situation is different. Under such conditions a HM-PEG polymer is likely to form a bridge between two microemulsion droplets. Since the latter results in a physically crosslinked network this is expected to have large consequences for the rheological properties of the system. At this stage a gel-like or highly viscous solution is expected. Fig. 3 shows both types of polymer organization, loops and bridges.

Generally, solutions only containing end-modified polymers are regarded to form polymeric micelles, sometimes referred to as flowers, at low concentrations. A large fraction of the HM-polymers within these flowers are in a loop conformation. This is mainly due to that the concentration of polymeric micelles is low, and the average distance between flowers is large. When the concentration increases the average distance between adjacent flowers decreases, and a bridge conformation becomes more likely. The bridges provide connectivity in the system, which gives rise

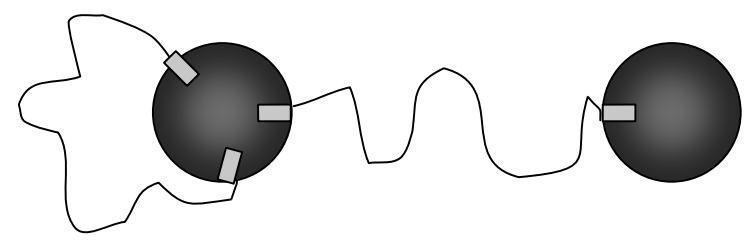

Fig. 3. Polymer-droplet association. Change over from loops to bridges can result from a change in microemulsion concentration. 
to an attraction between micelles [9,10]. Apart from favoring phase separation, the attraction also influences dynamics, and in particular the viscosity is expected to increase strongly as a result from network formation. We believe that the mechanism that is generally accepted to be responsible for the strong concentration dependency in solutions containing end-modified polymers [11], is closely related to what happens also in the present system. In an intermediate concentration range a transformation from loop to bridge conformations takes place.

In the present system, with microemulsion added, the Newtonian viscosity increases strongly as a function of microemulsion concentration, at low and intermediate concentrations, confirmed by Fig. 4, where is shown the Newtonian viscosity as a function of microemulsion concentration. Here it deserves to mention that strong concentration dependencies with high values of the power law exponent, $x\left(\eta=c^{x}\right)$ have also been found in semidilute systems of flexible polymers of high molecular weight under theta solvent conditions
[12], and in an aqueous solution containing only a HM-PEG polymer [13]. The large exponent was believed to be due to attraction between polymer chains as a result from poor solvent conditions. Here the microemulsion contains a fixed number of HM-PEG chains per microemulsion droplet, and the behavior is in sharp contrast to the weak concentration dependency that is observed for the same microemulsion in the same concentration range but without added HM-PEG. In the latter case, the concentration dependency of the viscosity is well represented by a hard sphere model [14].

Surprisingly it can also be seen in Fig. 4 that the increase in viscosity falls off at a further increase in the concentration of the microemulsion, and at even higher concentrations the viscosity decreases. This is unexpected since the viscosity normally is found to increase when the concentration increases. The reason to this behavior is 2-fold, first; the concentration of rheologically active links is expected to increase with concentration; and secondly it is normally found that the dynamics of a solution is slowed down at higher concentra-

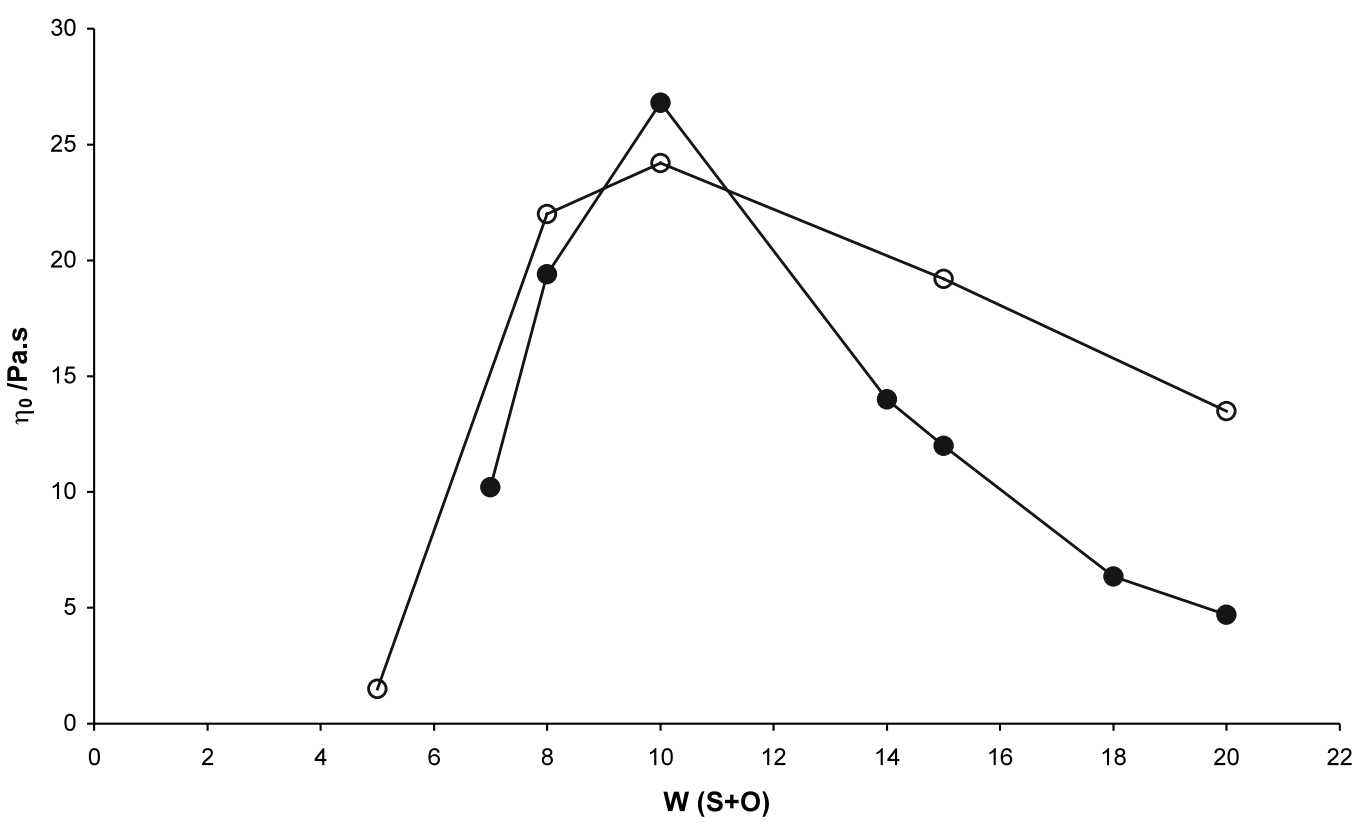

Fig. 4. Newtonian viscosity as a function of the microemulsion concentration at $25{ }^{\circ} \mathrm{C}$ at a fixed number of polymer molecules per microemulsion droplet $(\alpha=4)$. Initially the viscosity increases strongly as a function of microemulsion concentration for the $\mathrm{C}_{21} \mathrm{EO}_{280} \mathrm{C}_{21}$ polymer $(\mathrm{O})$, while at higher concentrations the viscosity falls off. $\mathrm{C}_{21} \mathrm{EO}_{450} \mathrm{C}_{21}(\boldsymbol{O})$ could not be investigated at a microemulsion concentration of $5 \mathrm{wt} . \%$ due to a phase separation. 
tions (due to entanglements etc.). However, a similar observation, with a decreased viscosity with an increasing concentration, has been reported in an aqueous solution containing only an end-capped PEG [15]. In the latter investigation the viscosity was found to decrease above a concentration of about $50 \mathrm{wt} . \%$ HM-PEG. This was suggested to be due to a gradual change, on the molecular level, from a structure containing micellelike aggregates inter-connected via polymer bridges to a more meltlike state, where microsegregation in hydrophilic and hydrophobic domains is less pronounced. A related mechanism may contribute also in the present solution. A stress-relaxation requires that the end-groups of the HM-PEG polymers move from one microemulsion droplet to another. The fact that the hydrophobic end-groups have to pass via an aqueous environment opposes this process. By increasing the microemulsion concentration the microemulsion droplets approach each other and the HM-PEG end-groups may have a possibility to move via the less polar palisade layers of the oil swollen micelles (these consist of ethylene oxide groups and water), which results in a higher dynamics and in a decrease in the viscosity.

The maximum in the viscosity is closely related to the interdroplet distance where the polymer chains are in their unperturbed states [16] (compare Fig. 4 and the detail in Fig. 11 which shows that above $\sim 11 \mathrm{wt} . \%$ microemulsion), the interdroplet length is lower than the unperturbed $\mathrm{C}_{21}-$ $\mathrm{PEG}-\mathrm{C}_{21}$. At higher droplet concentrations there will be an entropic conformational loss in forming a three-dimensional network and this unfavorable contribution translates into a decreased viscosity. In line with this we see a more important reduction in viscosity as the EO chain becomes longer.

When the droplets are close to each other the relaxation of the polymer chains becomes facilitated since a significant part of the volume between hydrocarbon domains is occupied by the EO chains; these have a lower polarity and thus the transfer of the hydrophobic end-caps becomes less unfavorable.

To test this hypothesis the dynamics that can be obtained from rheological measurements have to be investigated in detail. Fortunately the rheolo- gical response from the present system is expected to be rather simple since the low polymer concentration suggests that the contribution to dynamics from chain entanglements can be neglected $[8,13]$. Indeed, the Maxwell model that is the simplest model of a viscoelastic fluid gives a fair description of the dynamic moduli as a function of frequency.

$G_{\infty}$ and $\tau$ are obtained from this model (Eqs. (1a) and (1b)). The activation energy for the relaxation process was obtained from $\tau$ (Eq. (3)) in an Arrhenius' plot. An example is given in Fig. 5 , where is shown $\ln \tau$ as a function of the inverse of the temperature; $E / k_{\mathrm{B}}$ is given by the slope of the curve. The fact that straight lines are obtained in the Arrhenius' representation suggests that the structure of the solution is virtually unaffected in the investigated temperature range. This was the conclusion in a previous report where the same microemulsion and a similar HM-PEG polymer were investigated [8]. Since $E$ is likely to be

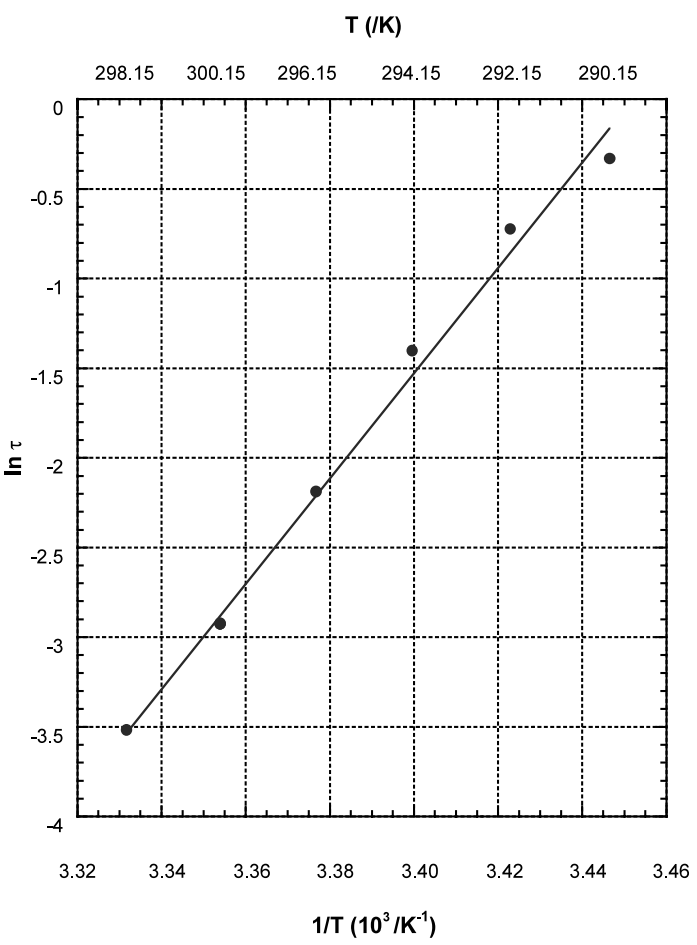

Fig. 5. Arrhenius plot of the temperature dependence of the relaxation time for a $10 \mathrm{wt} . \%$ microemulsion containing $\mathrm{C}_{21} \mathrm{EO}_{280} \mathrm{C}_{21}$. An apparent activation energy, $E$ of $106 k_{\mathrm{B}}$ was obtained. 
coupled to the process of transferring a polymer hydrophobic tail from a microemulsion droplet (oil environment) to an aqueous environment it is not surprising that the numbers obtained from the Arrhenius' plots are similar for polymers with the same hydrophobic end-groups, Table 2. From this it is also expected that the $\mathrm{C}_{24} \mathrm{E}_{X} \mathrm{C}_{24}$ polymers should have higher $E$ values than the $\mathrm{C}_{21} \mathrm{E}_{X} \mathrm{C}_{21}$ polymers have. This is indeed what is found, Table 2. The absolute values seem, however, to be very high; higher than would be expected if the temperature dependency of $\tau$ was only determined by the process where the hydrophobic tail of the HM-PEG polymer was transferred from an oil to an aqueous environment. The latter would give roughly $1 \mathrm{kT}$ per methylene group [17]. We will return to this observation below.

A masterplot performed by a superposition of mechanical spectra from different temperatures and by using the shift factors in Eqs. (4a) and (4b), is seen in Fig. 6.

Fig. 7(a) shows the evolution of $G_{\infty}$ with microemulsion concentration. It can be seen, as expected, that $G_{\infty}$ increases with concentration, also in the range where the viscosity was found to decrease. Regarding the evolution of $\tau$ in Fig. 7(b), we can see that $\tau$ has a more complex behavior and decreases strongly at higher concentrations. Since the Newtonian viscosity can be expressed as $[7,18,19]$.

$\eta_{\omega \rightarrow 0}^{*}=G_{\infty} \tau$

it is not surprising that the product of $G_{\infty}$ and $\tau$ follows the viscosity values presented in Fig. 4. The fact that $\tau$ decreases with an increasing concentration (above a certain microemulsion concentration) would suggest that the activation

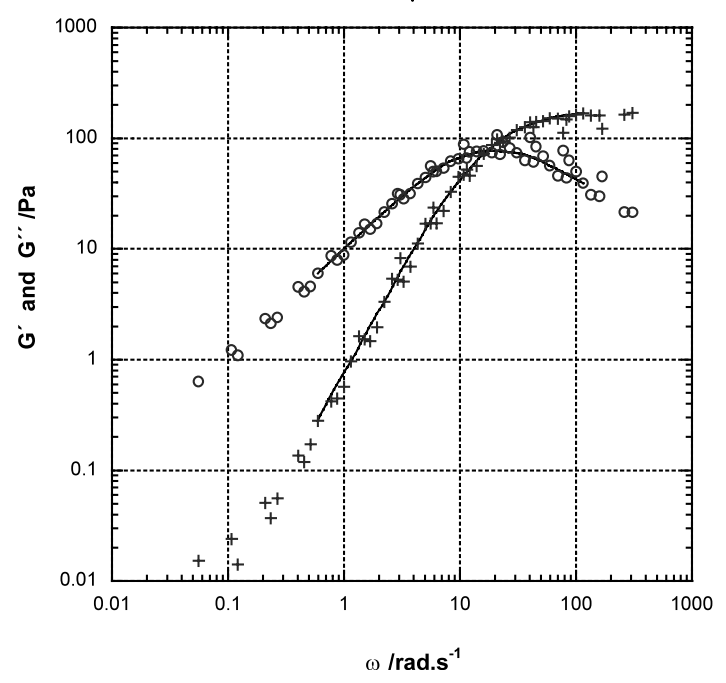

Fig. 6. Typical mechanical spectra of the frequency range which was extended by performing a superposition of the dynamic moduli from different temperatures by using Eqs. (4a) and (4b) and a reference temperature of $25{ }^{\circ} \mathrm{C}$. The measurements were performed on a $10 \mathrm{wt} . \%$ microemulsion to which the HM-P $\mathrm{C}_{24} \mathrm{EO}_{280} \mathrm{C}_{24}$ and Maxwell model did fit well to the experimental data.

energy, $E$, follows the same trend, Eq. (3). This is, however, not the case. In Fig. 8 is shown that $E$, oppositely to what is expected from the concentration dependency of $\tau$, actually increases with concentration.

Apart from the high numerical values of $E$ we now have two more observations that at first are unexpected ( $\tau$ decreases and $E$ increases with concentration).

It is well established that poly(ethylene oxide) chains repel each other at low temperatures. However, as temperature increases there is a progressively weaker repulsion that turns into an

Table 2

Summarizes activation energies (in units of $k_{\mathrm{B}} T$ ) in the different systems

\begin{tabular}{|c|c|c|c|c|}
\hline & $\mathrm{C}_{21} \mathrm{EO}_{280} \mathrm{C}_{21}$ & $\mathrm{C}_{24} \mathrm{EO}_{280} \mathrm{C}_{24}$ & $\mathrm{C}_{21} \mathrm{EO}_{450} \mathrm{C}_{21}$ & $\mathrm{C}_{24} \mathrm{EO}_{450} \mathrm{C}_{24}$ \\
\hline $5 \%$ microemulsion & 65 & & & \\
\hline $8 \%$ microemulsion & 95 & & 92 & \\
\hline $10 \%$ microemulsion & 106 & 117 & 100 & 115 \\
\hline $15 \%$ microemulsion & 140 & & 146 & \\
\hline $20 \%$ microemulsion & 155 & & 165 & \\
\hline
\end{tabular}



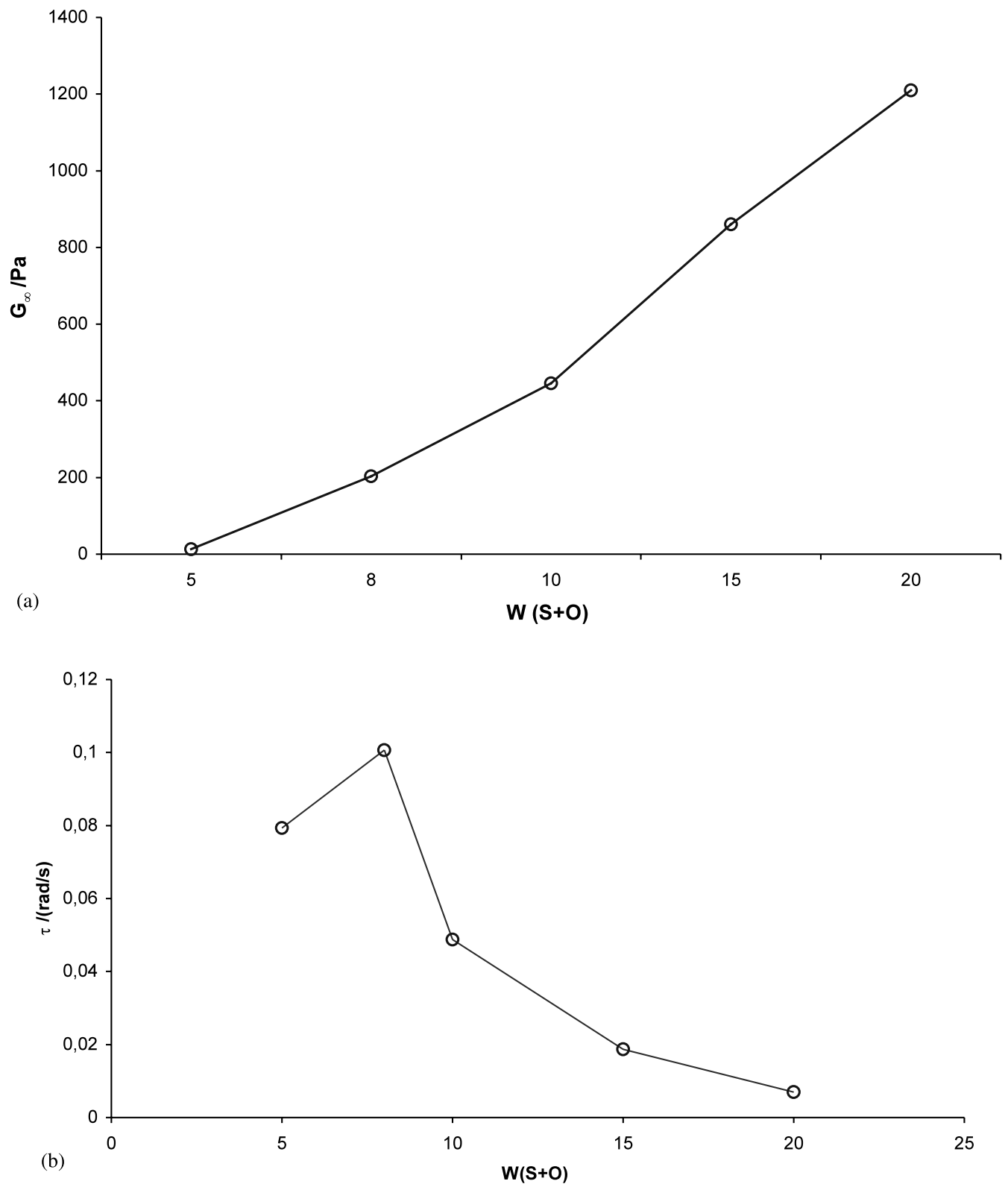

Fig. 7. (a) Illustrates the relation between the plateau of the storage modulus $\left(G_{\infty}\right)$ and the microemulsion concentration. It can be noted that $G_{\infty}$ increases with the microemulsion concentration, also in the range where the viscosity was found to decrease. (b) Shows the relation between the relaxation time $(\tau)$ and the microemulsion concentration. Compared with $G_{\infty}, \tau$ has a more complex behavior and decreases strongly at higher concentrations. Systems shown refer to $\mathrm{C}_{21} \mathrm{EO}_{280} \mathrm{C}_{21}$ at $25{ }^{\circ} \mathrm{C}$.

attraction [20]. This explains the strong temperature dependence and the high apparent $E$.

Strong temperature dependences can be seen at high microemulsion concentrations studied, as confirmed by Fig. 9(a and b), where is shown the relation between microemulsion concentration for several temperatures between 17 and $27{ }^{\circ} \mathrm{C}$. The droplets become more distant from each other 


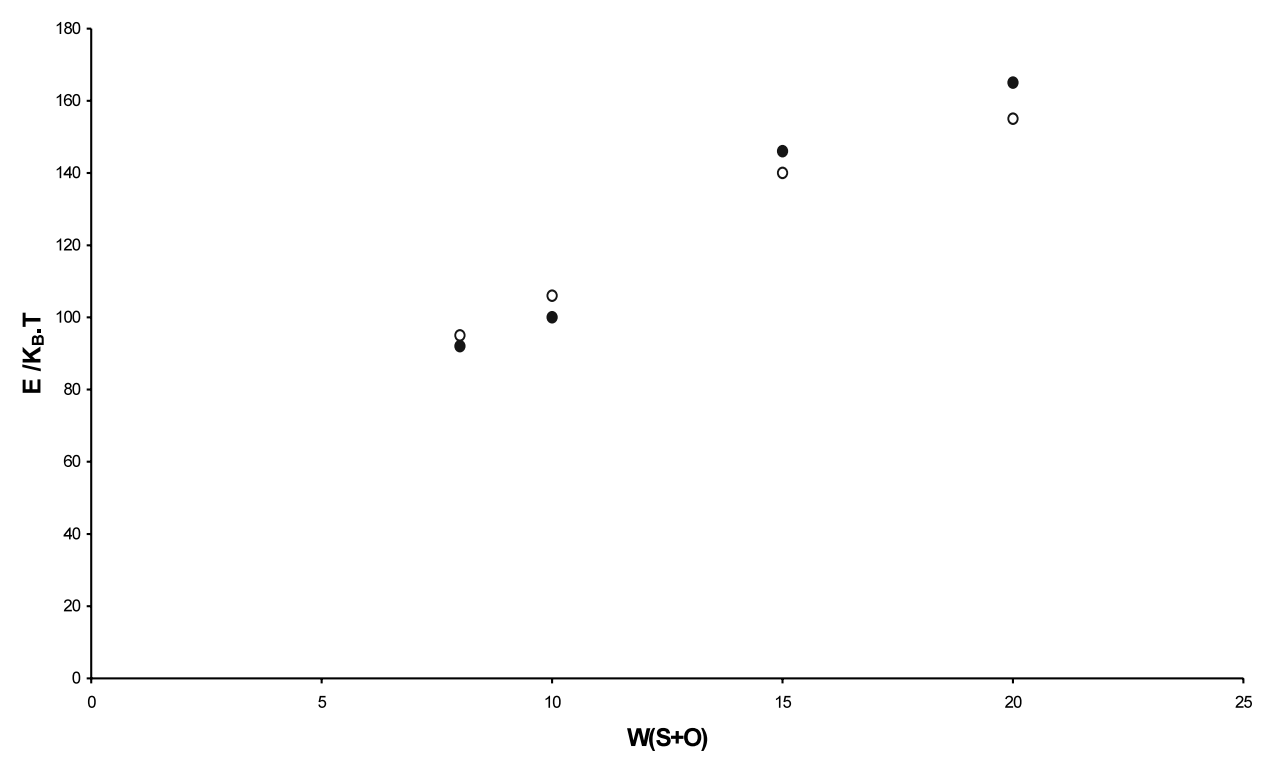

Fig. 8. The apparent activation energy, $E$, as a function of microemulsion concentration for microemulsions containing $\mathrm{C}_{21} \mathrm{EO}_{280} \mathrm{C}_{21}$ (O) or $\mathrm{C}_{21} \mathrm{EO}_{450} \mathrm{C}_{21}$ polymer $(\mathrm{O}$

when temperature decreases which induces a lower probability for the polymer chains to move to adjacent droplets; this increases the relaxation time and thus, the viscosity and the figures clearly denote a lower viscosity at high temperatures. The viscosity of long chain HM-PEG is more influenced by the temperature than the viscosity of the short one. This can be explained by the same arguments shown above for the concentration factor, considering that at high temperatures a facilitated droplet approach is expected.

As the temperature effect is more evident at high concentrations, the relaxation time becomes extremely low at high temperatures and high concentrations, which can explain the increase of $E$ with microemulsion concentration.

Concerning the concentration dependence of $\tau$ and $E$, we have above already explained that when the droplet volume fraction increases to very high values, the droplets are close enough so that transfer of alkyl end-caps does not have to proceed via a purely aqueous environment but via palisade layers rich in ethylene oxide groups. Since the palisade layers have a significantly lower polarity than water this facilitated transfer of alkyl chains between microemulsion droplets, reduces $\tau$. This transfer is for hard-sphere microemulsion droplets mainly determined by the volume fraction of droplets but will be modified if there is an attractive interaction.

In Fig. 10, the complex viscosity $\left(\eta^{*}=\right.$ $\sqrt{\left.\left(G^{\prime 2}+G^{\prime \prime 2}\right) / \omega^{2}\right)}$ is given as a function of $\omega$ for the four different polymers at a microemulsion concentration of $10 \%$. It can be seen that the data arranges in two groups. It seems that the viscosity, as it should, is only slightly influenced by the length of the mid-block of the HM-PEG polymer. Surprisingly the highest viscosity is obtained with the HM-PEG that has the shortest hydrophobic tail. This is, however, understood by observing that the polymers prepared with the shorter hydrocarbon tails give higher $G_{\infty}$ values, Table 3. Since ${ }^{1} \mathrm{H}-\mathrm{NMR}$ suggested the substitution degree to be about 1.2 (instead of the ideal 2.0) for all polymer fractions, we conclude that the synthesis has resulted in a larger fraction of disubstituted (and non-substituted) HM-PEG polymers with the shorter hydrophobic tail. The chemical reaction with the longer hydrophobic tail has on the other hand favored mono-substituted HM-PEG polymers. 

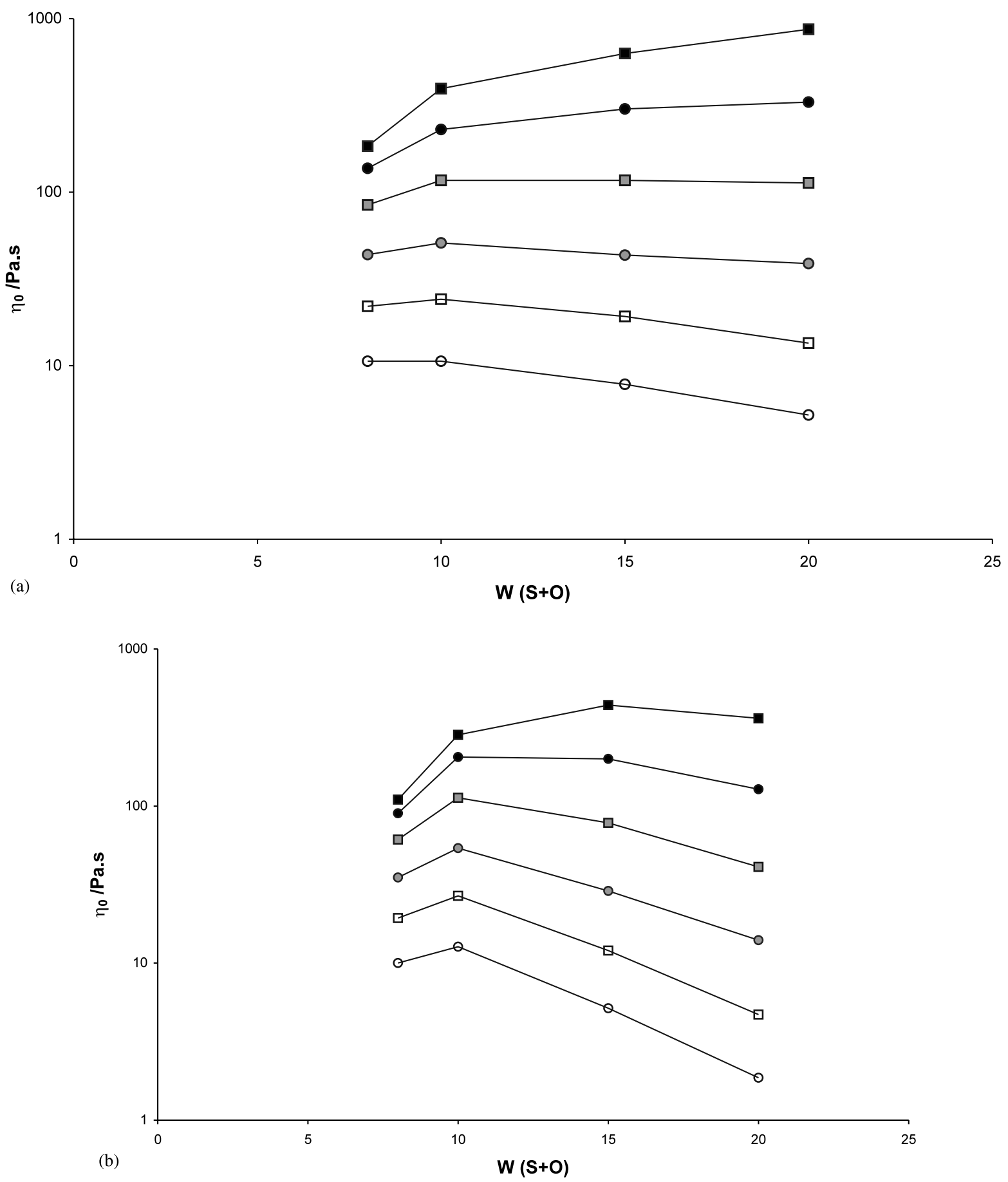

Fig. 9. The Newtonian viscosity $\left(\eta_{0}\right)$ as a function of the microemulsion concentration, $(W(\mathrm{~S}+\mathrm{O}))$, containing $\mathrm{C}_{21} \mathrm{EO}_{280} \mathrm{C}_{21}$ polymer;(9a) and $\mathrm{C}_{21} \mathrm{EO}_{450} \mathrm{C}_{21}$ polymer; (9b) for temperatures of $17{ }^{\circ} \mathrm{C}(\mathbf{\square}), 19{ }^{\circ} \mathrm{C}(\boldsymbol{\bigcirc}), 21{ }^{\circ} \mathrm{C}\left(\mathbb{8}^{4}\right), 23{ }^{\circ} \mathrm{C}\left(\mathbf{O}^{\circ}\right), 25{ }^{\circ} \mathrm{C}(\square)$ and $27{ }^{\circ} \mathrm{C}(\mathrm{O})$. 


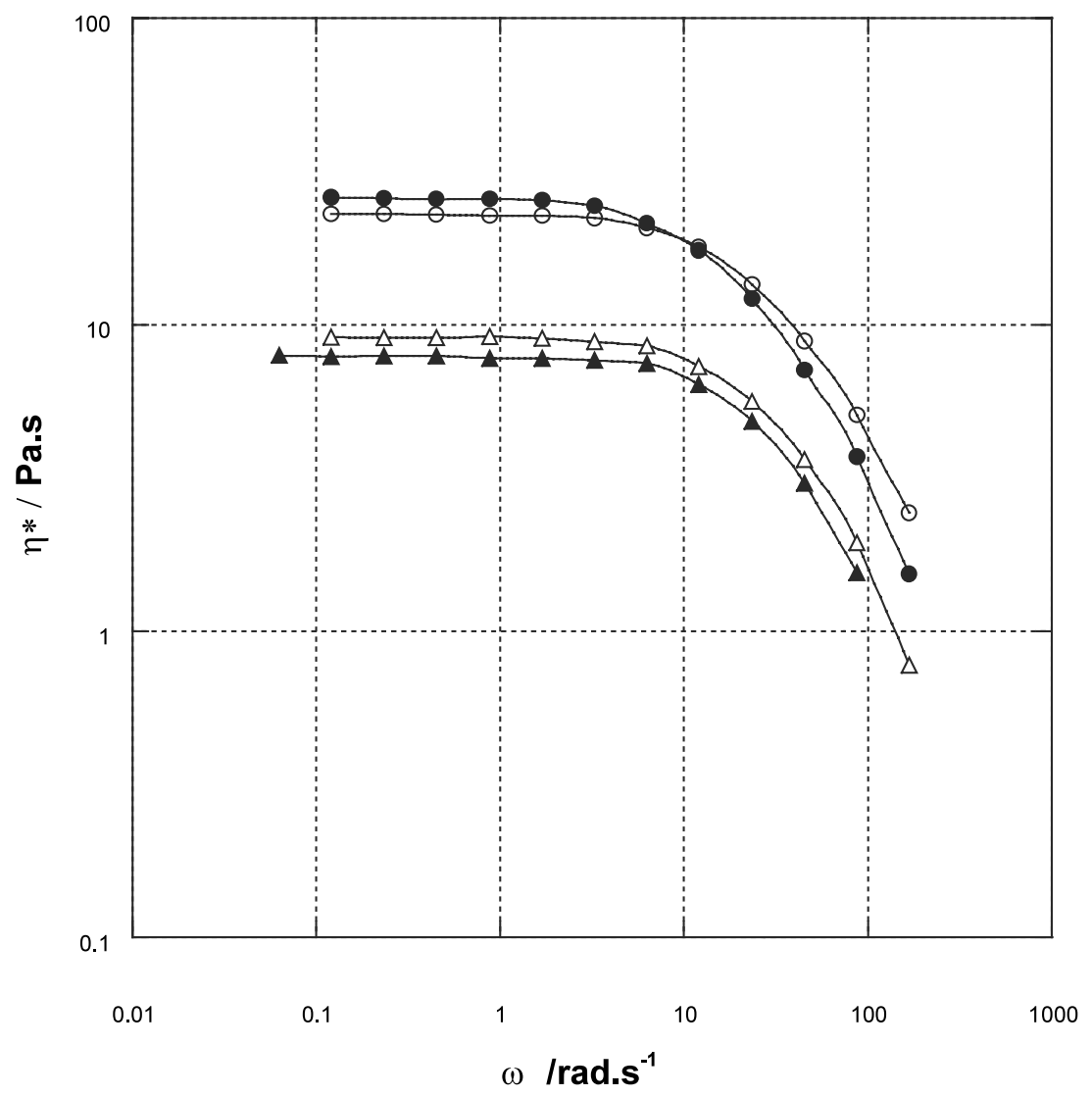

Fig. 10. The complex viscosity as a function of the angular frequency for $\mathrm{C}_{21} \mathrm{EO}_{280} \mathrm{C}_{21}(\bigcirc), \mathrm{C}_{21} \mathrm{EO}_{450} \mathrm{C}_{21}\left(\mathrm{O}_{)}, \mathrm{C}_{24} \mathrm{EO}_{280} \mathrm{C}_{24}(\Delta)\right.$ and

Table 3

Summarizes the fitting parameters $G_{\infty}$ and $\tau$ obtained when Maxwell elements were fitted to the rheological data for the four different polymers in the $10 \%$ microemulsion at $25{ }^{\circ} \mathrm{C}$

\begin{tabular}{lcccc}
\hline & $\mathrm{C}_{21} \mathrm{EO}_{280} \mathrm{C}_{21}$ & $\mathrm{C}_{24} \mathrm{EO}_{280} \mathrm{C}_{24}$ & $\mathrm{C}_{21} \mathrm{EO}_{450} \mathrm{C}_{21}$ & $\mathrm{C}_{24} \mathrm{EO}_{450} \mathrm{C}_{24}$ \\
\hline$G_{\infty}\left(\mathrm{Pa}^{-1}\right)$ & 451.59 & 175.45 & 338.74 & 124.84 \\
$\tau \times 10^{3}\left(\mathrm{~s}^{-1}\right)$ & 53.8 & 53.5 & 79.6 & 61.5 \\
\hline
\end{tabular}

Finally we have also roughly estimated how the fraction of rheologically active chains changes as a function of the average distance between microemulsion droplets. This is performed by using $G_{\infty}$ to calculate the variation in $n$ with Eq. (2) and relate it to the total polymer concentration. We have chosen to normalize the abscissa with the value obtained at the highest microemulsion concentration (20 wt.\%). (Actually, in a closely related system it has previously been concluded that at a concentration of $20 \mathrm{wt} . \%$ virtually all HM-PEG polymers that were bifunctional formed bridges [5].) From Fig. 11 it follows that the fraction of HM-PEG chains that give rheologically active bridges decreases more than a factor of 20 in the investigated regime (5-20 wt.\% microemulsion). It is possible that, as was discussed above, in this process the fraction of loop conformations increases since it seems unlikely that dangling ends form (due to unfavorable contact between the 


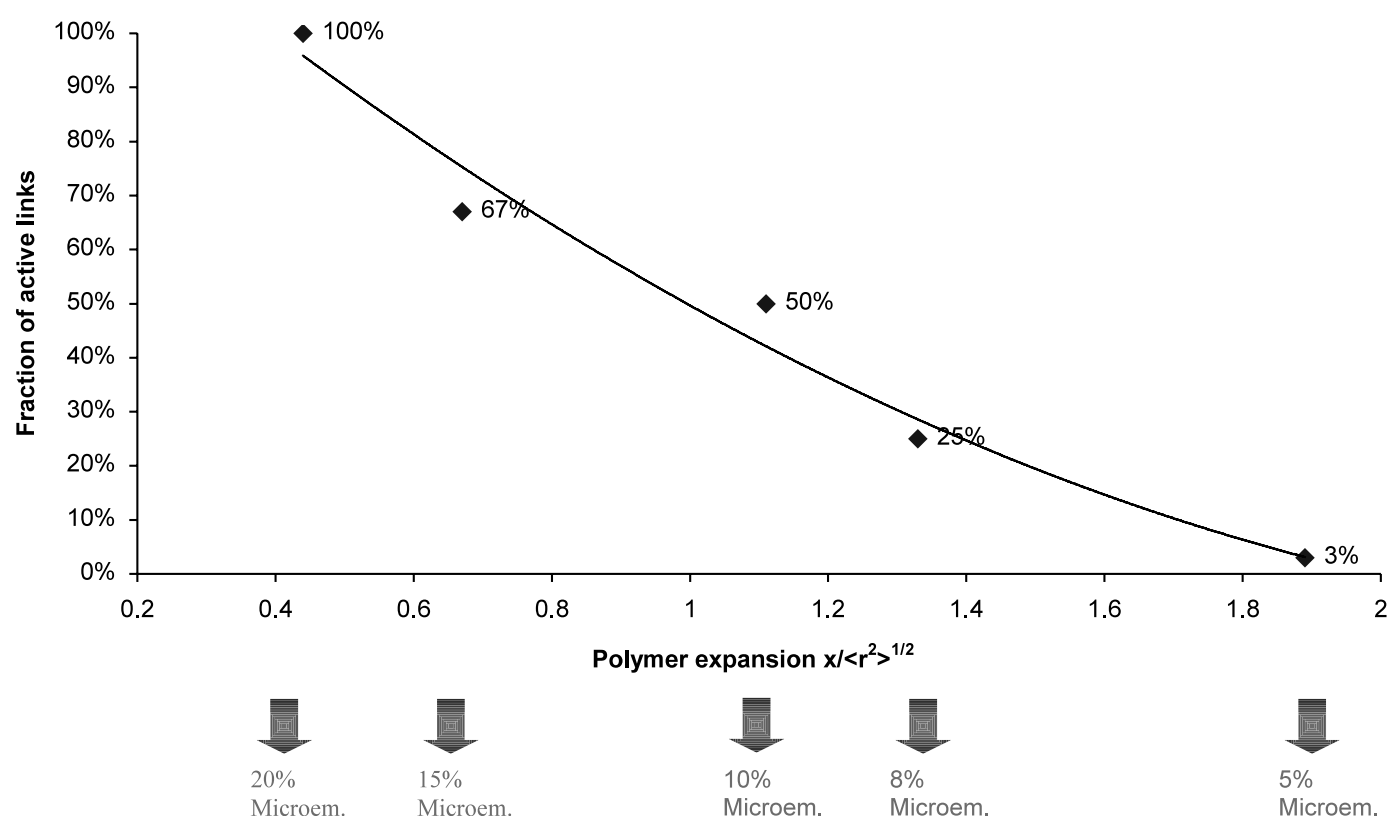

Fig. 11. The fraction of bridges, relative to concentration of bridges in $20 \mathrm{wt} . \%$ microemulsion, on dilution of the microemulsion. On the abscissa is given the end-to-end distance [9] of a PEG with the same molecular weight as of the mid-block of the HM-P $\mathrm{C}_{21} \mathrm{EO}_{280} \mathrm{C}_{21}$ divided by the average distance between the microemulsion droplets. When the abscissa value is lower than 1, the HM-P is compressed between adjacent droplets.

polymer hydrophobic tails and water). It could also be that dilution induces larger concentration fluctuations and domains enriched in microemulsion droplets form. These more concentrated domains are separated from each other with domains that have a lower concentration, and only held together by a smaller number of HMPEG chains. The latter seems a reasonable conclusion since microemulsions containing the $\mathrm{C}_{24} \mathrm{EO}_{x} \mathrm{C}_{24}$ polymer actually phase separated macroscopically upon dilution to $5 \mathrm{wt} . \% \mathrm{~A}$ dilute aqueous phase was formed in equilibrium with a viscous phase, which most likely contained the predominant amount of the microemulsion and the HM-PEG polymer. This observation is in line with other related investigations [8,21].

\section{Conclusions}

The addition of spherical $\mathrm{O} / \mathrm{W}$ microemulsion droplets with a small size distribution to the lowdisperse well-defined end-capped polymers can prevent, in the studied polymer concentration and temperature ranges, the known phase separation of aqueous solutions containing only HM-P. We have studied structure and dynamics of these systems by using rheology. It was found that the molecular association structure is sensitive to variations in the microemulsion concentration and to the length of the mid-block of the HMPEG polymers, while the characteristic relaxation time was sensitive to variations in the polymer hydrophobic moieties. The results could be understood by translating the microemulsion concentration to a mean distance between microemulsion droplets and comparing this length with the endto-end distance of the unmodified parent polymers. When the microemulsion concentration was decreased and the average distance between droplets became significantly longer than the end-end distance of the unmodified parent polymer a loopconformation was favored over a rheologically active link-formation. Apart from the concentration of active links that could be controlled by the microemulsion concentration and by the length of 
the HM-PEG mid-block, the viscosity also depends on the characteristic relaxation time of the system. This relaxation time was found to be strongly influenced by the length of the hydrophobic moiety of the HM-PEG polymers, the concentration of the microemulsion and by the temperature. To understand the maximum in the Newtonian viscosity as a function of microemulsion concentration, we need to focus first on the relaxation time of the process. This property decreases by increasing microemulsion concentration, giving a reduction in viscosity. At high microemulsion concentrations, the microemulsion droplets are closer and the polymer hydrophobic groups can shift between droplets by the less polar palisade layers. This process facilitates relaxation.

Temperature will have special effects on relaxation because of the anomalous temperature dependence of the interaction between oxyetylene chains and water [17]. Due to conformational changes to less polar states on increasing the temperature, the oxyethylene chain-water interaction will become less favorable. This will effect both the polymer and the surfactant molecules. In particular the microemulsion droplets will change from being repulsive at low temperature to attractive at higher temperature. This will lead to droplet clustering and a facilitated transfer of polymer hydrophobes between droplet. This will contribute to the strongly decreased relaxation time at higher temperatures.

\section{Acknowledgements}

We thank Leif Karlson at Akzo Nobel Surface Chemistry, Stenungsund, Sweden, for supplying HM-PEG polymers and for valuable discussions. KT also thanks the Center for Amphiphilic Polymers (CAP) at Lund University for financial support. The stay of FA in Lund was financed from a grant from the Swedish Science Research Council. The Colloid Group in Coimbra Univer- sity is supported by a grant from the Fundação para Ciência e Tecnologia (FCT) (project Sapiens PCTI/99/QUI/35415).

\section{References}

[1] J.E. Glass (Ed.), Polymers in Aqueous Media, vol. 223, American Chemical Society, Washington, DC, 1989.

[2] U. Olsson, P. Schurtenberger, Langmuir 9 (1993) 3389.

[3] E. Michel, M. Filali, R. Aznar, G. Porte, J. Appell, Langmuir 16 (2000) 8702.

[4] M. Filali, R. Aznar, M. Svenson, G. Porte, J. Appell, J. Phys. Chem. B 103 (1999) 7293.

[5] M. Filali, M. Ouazzani, E. Michel, R. Aznar, G. Porte, J. Appell, J. Phys. Chem. B 105 (2001) 10528.

[6] H. Bagger-Jörgensen, U. Olsson, K. Mortensen, Langmuir 13 (1997) 1413-1421.

[7] M.S. Green, A.V.J. Tobolsky, Chem. Phys. 14 (1946) $80-$ 92.

[8] H. Bagger-Jörgensen, L. Coppola, K. Thuresson, U. Olsson, K. Mortensen, Langmuir 13 (1997) 4204-4218.

[9] A.N. Semenov, J.-F. Joanny, A.R. Khokhlov, Macromolecules 28 (1995) 1066-1075.

[10] T.A.J. Witten, Physique 49 (1988) 1055-1063.

[11] M.A. Winnik, A. Yekta, Curr. Opin. Colloid Interf. Sci. 2 (1997) 424-436.

[12] Y. Takahashi, Y. Isono, I. Noda, M. Nagasawa, Macromolecules 18 (1985) 1002.

[13] K. Thuresson, S. Nilsson, A.-L. Kjøniksen, H. Walderhaug, B. Lindman, B. Nyström, J. Phys. Chem. B 103 (1999) 1425-1436.

[14] M. Leaver, U. Olsson, Langmuir 10 (1994) 3449-3454.

[15] L. Karlson, S. Nilsson, K. Thuresson, Colloid Polym. Sci. 277 (1999) 798-804.

[16] P. Gregory, M.B. Huglin, Makromol. Chem. 187 (1986) $1745-1755$.

[17] C. Tanford, The Hydrophobic Effect: Formation of Micelles and Biological Membranes, second ed., Wiley, New York, 1980.

[18] M. Doi, S.F. Edwards, The Theory of Polymer Dynamics, first ed., Oxford University Press, New York, 1986.

[19] F. Tanaka, S.F. Edwards, J. Non-Newtonian Fluid Mechanics 43 (1992) 247-271.

[20] B. Jönsson, B. Lindman, K. Holmberg, B. Kronberg, Surfactants and Polymers in Aqueous Solution, Wiley, London, 1997.

[21] K. Thuresson, F. Joabsson, Colloids Surf., A: Physicochem. Eng. Aspects 151 (1999) 513-523. 